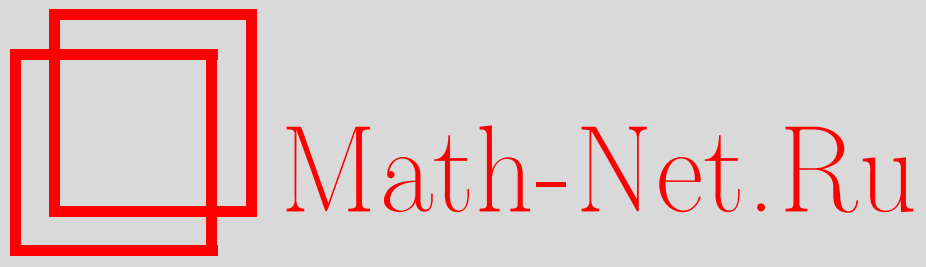

С. В. Облезин, Дискретные симметрии систем изомонодромных деформаций дифференциальных уравнений второго порядка фуксова типа, Функи. анализ и его прил., 2004, том 38, выпуск 2, 38-54

DOI: https://doi.org/10.4213/faa106

Использование Общероссийского математического портала MathNet.Ru подразумевает, что вы прочитали и согласны с пользовательским соглашением

http://www . mathnet.ru/rus/agreement

Параметры загрузки:

IP : 54.198 .55 .26

26 апреля 2023 г., 14:58:07

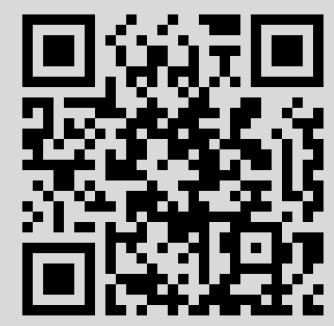




\title{
Дискретные симметрии систем изомонодромных деформаций дифференциальных уравнений второго порядка фуксова типа*
}

\author{
(c) 2004. С. В. ОБЛЕзин
}

\section{§1. Введение}

В данной работе изучаются дискретные преобразования пространств модулей логарифмических $s l(2)$-связностей с особенностями в различных точках $\left\{x_{1}, \ldots, x_{n}\right\}$ на сфере Римана $\mathbb{P}^{1}$ с фиксированными собственными значениями вычетов связности. Основным результатом является вычисление групповой структуры изоморфизмов между этими пространствами модулей. Всякая такая связность задает некоторое дифференциальное уравнение с регулярными особенностями на $\mathbb{P}^{1}$; при этом вычеты связности соответствуют локальным параметрам решений уравнения. Мы рассматриваем дискретные преобразования параметров этого уравнения, не меняющие локальные монодромии его решений. Можно переформулировать задачу следующим образом. Рассмотрим дифференциальное уравнение второго порядка с регулярными особенностями в точках $\left\{x_{1}, \ldots, x_{n}\right\}$ на сфере Римана и включим его в изомонодромное аналитическое семейство - для фундаментальной матрицы решений нашего уравнения $Y(z)$ рассмотрим систему

$$
\partial_{z} Y(z) \cdot Y(z)^{-1}=\sum_{i} \frac{B_{i}\left(x_{1}, \ldots, x_{n}\right)}{z-x_{i}}
$$

с условием совместности

$$
d B_{i}\left(x_{1}, \ldots, x_{n}\right)=\sum_{j}\left[B_{j}, B_{i}\right] d \log \left(x_{i}-x_{j}\right),
$$

которое называется уравнением Шлезингера; далее, зададим начальные условия этой изомонодромной деформации конфигурации $n$ точек на $\mathbb{P}^{1}$. Такая деформация называется системой Шлезингера, и дискретные симметрии этой системы можно понимать как ее дискретные времена. Пространство начальных данных системы Шлезингера является грубым пространством модулей $\mathscr{M}_{n}$ наборов $\left(\mathscr{L}, \nabla, \phi ; \lambda_{1}, \ldots, \lambda_{n}\right)$, где $\mathscr{L}$ есть расслоение ранга 2 на $\mathbb{P}^{1}$ со связностью $\nabla: \mathscr{L} \rightarrow \mathscr{L} \otimes \Omega_{\mathbb{P}^{1}}^{1}\left(x_{1}+\cdots+x_{n}\right)$, а $\phi: \operatorname{det} \mathscr{L} \simeq \mathscr{O}_{\mathbb{P}^{1}}$ - горизонтальный изоморфизм; при этом вычеты связности $\nabla$ в точках $x_{i}, i=1, \ldots, n$, имеют собственные значения $\left(\lambda_{i},-\lambda_{i}\right)$.

Для вычисления описанной группы дискретных преобразований в $\S \S 2$ и 3 мы развиваем геометрическую технику модификаций векторных расслоений со

* Данная работа была выполнена при частичной поддержке Программы Президента РФ по поддержке ведущих научных школ (грант НШ-1999.2003.2), гранта РФФИ 01-01-00539 и гранта CRDF RM1-2545. 
связностью [1]. В оригинальной работе [19] Шлезингер рассмотрел такие преобразования систем изомонодромных деформаций; с современной точки зрения алгебраические аспекты преобразований Шлезингера рассмотрены в работе Джимбо и Мивы [15] (см. также [16]), однако без изучения групповой структуры. Кроме того, в этих классических работах обсуждается представление монодромии в группе $G L(N)$. В настоящей работе мы рассматриваем $S L(2)$ представление, легко обобщаемое на классический случай $G L(2)$; между тем групповая структура в $S L$-случае оказывается более интересной и богатой. Следуя работе Аринкина и Лысенко [1], мы даем геометрическую интерпретацию и описываем преобразования Шлезингера как модификации расслоений со связностью. Нашим основным инструментом является конструкция $F$-пучков, или модификаций расслоений. Впервые модификации появились в работах Г. Биркгофа (см. [3]) в качестве сингулярных калибровочных преобразований в задаче Римана-Гильберта для интегральных уравнений. Затем в 30-е годы модификации появились в работах Э. Гекке (см. [10]), и теперь их называют соответствиями Гекке между модулярными кривыми. В это же время А. Вейль использовал конструкцию $F$-пучков для описния классов матричных дивизоров на кривой и обобщения теории Абеля-Якоби [23]. В работе [6] рассматриваются $F$-пучки для произвольного глобального поля; эта конструкция используется для доказательства глобальной гипотезы Ленглендса для $G L(2)$. Такие пучки называются пучками Фробениуса-Гекке или «штуками». В последнее время модификации широко используются в математической физике и геометрической теории представлений (см., например, $[1,22,8,14,17])$.

Наша группа преобразований пространства модулей $\mathscr{M}_{n}$ оказывается изоморфной аффинной группе Вейля системы корней типа $C_{n}$

$$
\mathfrak{W}\left(\widehat{C}_{n}\right) \simeq T \rtimes\left((\mathbb{Z} / 2 \mathbb{Z})^{n} \rtimes \mathfrak{S}_{n}\right)
$$

для $n$ регулярных особенностей $x_{i}$. В терминах нашей исходной задачи структура группы описывается следующим образом. Группа $\mathfrak{S}_{n}$ порождается перестановками особых точек, которые представляются изоморфизмами пространств начальных данных изомонодромной деформации

$$
\begin{aligned}
\mathscr{M}_{n}(\mathscr{L}, \nabla ; \phi: \operatorname{det} \mathscr{L} \simeq \mathscr{O} ; & \left.\lambda_{1}, \ldots \lambda_{i}, \ldots, \lambda_{j}, \ldots, \lambda_{n}\right) \\
& \simeq \mathscr{M}_{n}\left(\mathscr{L}, \nabla ; \phi: \operatorname{det} \mathscr{L} \simeq \mathscr{O} ; \lambda_{1}, \ldots, \lambda_{j}, \ldots, \lambda_{i}, \ldots, \lambda_{n}\right) .
\end{aligned}
$$

Трансляционная часть $T$ аффинной группы действует сдвигами собственных значений вычетов связности, причем $T$ содержит «короткие» и «длинные» сдвиги; эти сдвиги представляют изоморфизмы

$$
\begin{aligned}
\mathscr{M}_{n}(\mathscr{L}, \nabla ; \phi: \operatorname{det} \mathscr{L} & \left.\simeq \mathscr{O} ; \lambda_{1}, \ldots, \lambda_{n}\right) \\
& \simeq \mathscr{M}_{n}\left(\mathscr{L}, \nabla ; \phi: \operatorname{det} \mathscr{L} \simeq \mathscr{O} ; \lambda_{1}, \ldots, \lambda_{i}+\frac{1}{2}, \ldots, \lambda-\frac{1}{2}, \ldots, \lambda_{n}\right)
\end{aligned}
$$

И

$\mathscr{M}_{n}\left(\mathscr{L}, \nabla ; \phi: \operatorname{det} \mathscr{L} \simeq \mathscr{O} ; \lambda_{1}, \ldots, \lambda_{n}\right) \simeq \mathscr{M}_{n}\left(\mathscr{L}, \nabla ; \phi: \operatorname{det} \mathscr{L} \simeq \mathscr{O} ; \lambda_{1}, \ldots, \lambda_{k}+1, \ldots, \lambda_{n}\right)$ соответственно. Кроме этого можно менять знаки собственных значений вычетов связности в особых точках, т. е. применять элементы второго порядка

$$
\sigma^{i}:\left(\begin{array}{cc}
\lambda_{i} & 0 \\
0 & -\lambda_{i}
\end{array}\right) \rightarrow\left(\begin{array}{cc}
-\lambda_{i} & 0 \\
0 & \lambda_{i}
\end{array}\right)
$$


группы Вейля $s l(2)$-орбит вычетов связности в каждой точке $x_{i}$. Эти преобразования образуют нормальную подгруппу $(\mathbb{Z} / 2 \mathbb{Z})^{n}$ конечной факторгруппы $\mathfrak{W}\left(C_{n}\right)$; элемент $\left(\epsilon_{1}, \ldots, \epsilon_{n}\right) \in(\mathbb{Z} / 2 \mathbb{Z})^{n}$ представляет изоморфизм

$$
\mathscr{M}_{n}\left(\mathscr{L}, \nabla ; \phi: \operatorname{det} \mathscr{L} \simeq \mathscr{O} ; \lambda_{1}, \ldots, \lambda_{n}\right) \simeq \mathscr{M}_{n}\left(\mathscr{L}, \nabla ; \phi: \operatorname{det} \mathscr{L} \simeq \mathscr{O} ; \epsilon_{1} \lambda_{1}, \ldots, \epsilon_{n} \lambda_{n}\right) .
$$

Если взять композицию пары модификаций в различных точках с парой локальных транспозиций $\sigma_{i}, \sigma_{j}$ или пару модификаций в одной точке с транспозицией $\sigma_{i}$, то получатся элементы конечного порядка, отличные от перестановок; кроме того, можно получить элемент конечного порядка, скомбинировав пару модификаций в различных точках с перестановкой этих точек. Такие композиции описывают структуру полупрямого произведения групп. Трансляционная часть $T$ изоморфна целочисленной решетке $C_{n}$ ранга $n$, которая в стандартном базисе $\{e\}=\delta_{i j} n$-мерного векторного пространства представляется в виде

$$
C_{n}=\left\langle \pm 2 e_{i}, \pm e_{i} \pm e_{j}\right\rangle
$$

Таким образом, наша группа преобразований является группой автоморфизмов целочисленной решетки в $\mathbb{R}^{n}$.

В $\S \S 4,5$ и 6 полученный результат анализируется на нескольких классических примерах дифференциальных уравнениях фуксова типа - гипергеометрического уравнения (см. классические справочники $[2,13])$, уравнения Гойна $[11,7,2]$ и шестого уравнения Пенлеве $[18,12,9,1]$. В этом смысле данную часть работы можно воспринимать как по возможности наиболее полный библиографический обзор классических работ о симметриях фуксовых дифференциальных уравнений с тремя и четырьмя особенностями $[2,13]$; подобные вычисления для уравнений с бо́льшим числом фуксовых особенностей найти в цитируемой литературе не удалось.

Если фуксово дифференциальное уравнение второго порядка имеет три особых точки на $\mathbb{P}^{1}$, то заменами его можно привести к гипергеометрическому уравнению. Группа $\mathfrak{W}\left(\widehat{C}_{3}\right)$ его дискретных симметрий была полностью изучена К. Гауссом и Э. Куммером; наше изложение соответствует книге [2].

Далее излагается случай фуксова уравнения второго порядка с четырьмя особенностями, называемого уравнением Гойна [2]. Соответствующие вычисления дискретных симметрий были проделаны Гойном [11] и представляют собой 192 соотношения на решения, аналогичные двадцати четырем соотношениям Куммера для гипергеометрических функций.

Как объяснялось выше, можно включить уравнение Гойна в изомонодромное аналитическое семейство, т. е. написать деформацию Шлезингера этого уравнения. В частном случае системы с четырьмя фуксовыми особенностями с $s l(2)$-монодромиями такие вычисления приведут к шестому уравнению Пенлеве $P_{\mathrm{VI}}$. Его симметрии подробно изучались в работах $[18,12,1]$; группа симметрий этого уравнения изоморфна $\mathfrak{W}\left(\widehat{F}_{4}\right)$, т. е. является расширением группы $\mathfrak{W}\left(\widehat{D}_{4}\right)$ с помощью группы $\mathfrak{S}_{3}$ автоморфизмов графа $D_{4}$.

Я выражаю глубокую признательность и благодарность А. М. Левину за постановку задач и за многочисленные обсуждения данной работы. Я благодарен М. А. Ольшанецкому, а также А. В. Зотову, В. П. Лексину и В. Н. Рубцову за внимание и интерес, проявленные к этой работе, и ряд полезных замечаний. Также хочу поблагодарить В. Побережного за обсуждение действий особых калибровочных преобразований на регулярные системы, изложенных в начале 44 . 


\section{§2. Модификации расслоений ранга $N$ со связностями}

Рассмотрим расслоение $\mathscr{L}$ ранга $N$ со связностью $\nabla$ и точку $x$ на базе $\mathbb{P}^{1}$. Далее, обозначим слой в этой точке через $V:=\mathscr{L}_{x}$ и выберем в нем $k$-мерное подпространство $U \subset V$. Отождествляя расслоение $\mathscr{L}$ с пучком его сечений, рассмотрим следующие его модификации:

$$
(x, U)^{\text {low }} \mathscr{L}:=\{s \in \mathscr{L} \mid s(x) \in U\}, \quad(x, U)^{\text {up }} \mathscr{L}:=(x, U)^{\text {low }}(\mathscr{L}) \otimes \mathscr{O}(x) ;
$$

будем их называть нижней и верхней модификациями соответственно. Обозначим модифицированное расслоение $(x, U)^{\text {low }}(\mathscr{L})$ через $\widetilde{\mathscr{L}}$; тогда образом естественного вложения $\widetilde{\mathscr{L}}_{x} \rightarrow \mathscr{L}_{x}$ будет как раз $U$. В то же время, если $\widetilde{U}:=\operatorname{ker}\left(\widetilde{\mathscr{L}}_{x} \rightarrow \mathscr{L}_{x}\right)$, то $(x, \widetilde{U})^{\text {uр }} \widetilde{\mathscr{L}}=\mathscr{L}$. По-другому модификации можно описать точными тройками пучков

$$
\begin{gathered}
0 \rightarrow(x, U)^{\text {low }}(\mathscr{L}) \rightarrow \mathscr{L} \rightarrow \delta_{x} \otimes \mathscr{L}_{x} / U \rightarrow 0, \\
\left.0 \rightarrow \mathscr{L} \rightarrow(x, U)^{\text {up }} \mathscr{L} \rightarrow \delta_{x} \otimes U \otimes \mathscr{O}(x)\right|_{x} \rightarrow 0
\end{gathered}
$$

соответственно, где $\delta_{x}$ обозначает пучок-небоскреб с носителем в точке $x$. Грубо говоря, если предположить, что для расслоения $\mathscr{L} \simeq V \otimes \mathscr{O}$ имеется глобальное разложение $V=U \oplus \widetilde{U}$, то

$$
(x, U)^{\text {low }}(\mathscr{L})=U \otimes \mathscr{O} \oplus \widetilde{U} \otimes \mathscr{O}(-x), \quad(x, U)^{\mathrm{up}}(\mathscr{L})=U \otimes \mathscr{O}(x) \oplus \widetilde{U} \otimes \mathscr{O} .
$$

Другими словами, мы модифицируем расслоение, меняя базис сечений

$$
\left\{s_{1}(z), \ldots, s_{N}(z)\right\}
$$

в окрестности точки $x$ в следующем смысле. Если в этом локальном базисе

$$
U \otimes \mathscr{O} \simeq\left\{s_{1}(z), \ldots, s_{k}(z)\right\} \quad \text { и } \quad \widetilde{U} \otimes \mathscr{O} \simeq\left\{s_{k+1}(z), \ldots, s_{N}(z)\right\},
$$

то базис нижней модификации расслоения порождается сечениями

$$
\left\{s_{1}(z), \ldots, s_{k}(z),(z-x) s_{k+1}(z), \ldots,(z-x) s_{N}(z)\right\},
$$

а базис верхней модификации - сечениями

$$
\left\{(z-x)^{-1} s_{1}(z), \ldots,(z-x)^{-1} s_{k}(z), s_{k+1}(z), \ldots, s_{N}(z)\right\} .
$$

Иначе говоря, в проколотой окрестности действие таких модификаций можно представлять следующими матрицами переклейки:

$$
(x, U)^{\text {low }}=\left(\begin{array}{cc}
\mathbf{1}_{k} & 0 \\
0 & (z-x) \cdot \mathbf{1}_{N-k}
\end{array}\right), \quad(x, U)^{\text {up }}=\left(\begin{array}{cc}
(z-x)^{-1} \mathbf{1}_{k} & 0 \\
0 & \mathbf{1}_{N-k}
\end{array}\right),
$$

где через $\mathbf{1}_{m}$ обозначена единичная $(m \times m)$-матрица. Теперь рассмотрим связности с особенностями и их модификации; для этого нам понадобится следующее

ОПРЕДЕЛЕНИЕ [20]. Модулем $\mathfrak{M}$ с носителем $S$ на алгебраической кривой $X$ назовем конечный набор точек $S=\left\{x_{1}, \ldots, x_{n}\right\} \subset X$ и функцию, сопоставляющую каждой точке $x_{i}$ целое положительное число $n_{i}$. При необходимости мы будем отождествлять $\mathfrak{M}$ с эффективным дивизором $\sum n_{i} \cdot x_{i}$; нас интересует модуль $\mathfrak{M}=\sum x_{i}$. 
Итак, рассмотрим расслоение $\mathscr{L}$ со связностью $\nabla: \mathscr{L} \rightarrow \mathscr{L} \otimes \Omega^{1}(\mathfrak{M})$ и обсудим действие модификаций на такой связности. Сначала заметим, что модификация расслоения $\mathscr{L}$ в точке $x \in \mathbb{P}^{1}$ меняет детерминант:

$\operatorname{det}(x, U)^{\text {low }} \mathscr{L}=\operatorname{det} \mathscr{L} \otimes \mathscr{O}(-x \cdot \operatorname{dim} V / U), \quad \operatorname{det}(x, U)^{\text {up }} \mathscr{L}=\operatorname{det} \mathscr{L} \otimes \mathscr{O}(x \cdot \operatorname{dim} U)$.

Выберем в нашем расслоении связность $\nabla$ с логарифмической особенностью в точке $x$, и пусть подпространство $U \subseteq V:=\mathscr{L}_{p}$ будет инвариантным относительно вычета $\operatorname{Res}_{x} \nabla$, т. е. $\left(\operatorname{Res}_{x} \nabla\right)(U) \subseteq U$. Модифицируем расслоение в этом подпространстве. Рассмотрим, например, нижнюю модификацию $\widetilde{\mathscr{L}}$ со связностью

$$
\nabla^{\prime}: \widetilde{\mathscr{L}} \stackrel{\left.\nabla\right|_{\widetilde{\mathscr{L}}}}{\longrightarrow} \mathscr{L} \otimes \Omega^{1}(\mathfrak{M}) \stackrel{\text { pr }}{\longrightarrow} \widetilde{\mathscr{L}} \otimes \Omega^{1}(\mathfrak{M})
$$

тогда на детерминанте модифицированного расслоения получится связность

$$
\operatorname{tr} \nabla^{\prime}=\operatorname{tr} \nabla+\frac{d z}{z-x} \cdot \operatorname{dim} U
$$

Важно понимать, что пара «расслоение со связностью» модифицируется именно в $\left(\operatorname{Res}_{x} \nabla\right)$-инвариантных подпространствах; в противном случае связность может увеличить порядок полюса в особой точке. В самом деле, пользуясь матричным представлением, запишем действие модификации в неинвариантном подпространстве на связность в особой точке $x=0$ :

$$
\left(\begin{array}{ll}
1 & 0 \\
0 & z
\end{array}\right)\left[d+\left(\begin{array}{cc}
\lambda / z & \epsilon / z \\
0 & -\lambda / z
\end{array}\right)\right]\left(\begin{array}{cc}
1 & 0 \\
0 & 1 / z
\end{array}\right)=d+\left(\begin{array}{cc}
\lambda / z & \epsilon / z^{2} \\
0 & -(\lambda+1) / z
\end{array}\right)
$$

где $z$ - локальный параметр.

В дальнейшем нас будут интересовать расслоения ранга 2 ; поэтому мы вполне обойдемся лишь модификациями в одномерных инвариантных подпространствах, т.е. положим $\operatorname{dim} U=1$. Более того, мы будем рассматривать пары «верхняя модификация - нижняя модификация» в точках $x_{i}$ и $x_{j}$, чтобы получать расслоения $\mathscr{L}^{\prime \prime}$ с исходным детерминантом: $\operatorname{det} \mathscr{L}^{\prime \prime}=\operatorname{det} \mathscr{L} \otimes \mathscr{O}\left(x_{j}-x_{i}\right) \simeq$ $\operatorname{det} \mathscr{L} ;$ для этого мы, кроме того, зафиксируем набор совместных изоморфизмов $\mathscr{O} \simeq \mathscr{O}\left(x_{i}-x_{j}\right)$, таких, что

$$
\mathscr{O} \simeq \mathscr{O}\left(x_{i}-x_{j}\right) \otimes \mathscr{O}\left(x_{j}-x_{k}\right) \simeq \mathscr{O}\left(x_{i}-x_{k}\right) .
$$

Наконец, если мы применим эту конструкцию к расслоению $\mathscr{L}$ ранга 2 с $s l(2)$-связностью $\nabla$, то результатом будет расслоение $\mathscr{L}^{\prime \prime}$ со связностью

$$
\nabla^{\prime \prime}=\nabla+P_{U_{i}} \frac{d z}{z-x_{i}}-P_{\widetilde{U}_{j}} \frac{d z}{z-x_{j}},
$$

где $P_{*}$ обозначает проекторы на соответствующие $\operatorname{Res} \nabla$-инвариантные подпространства. Однако это уже не $s l(2)-$, а $g l(2)$-связность, и чтобы получить снова $s l(2)$-связность, нужно ее подправить на подходящую 1-форму:

$$
\widetilde{\nabla}^{\prime \prime}=\nabla^{\prime \prime}+\frac{1}{2}\left(\frac{d z}{z-x_{j}}-\frac{d z}{z-x_{i}}\right) .
$$

Таким образом, мы построили нетривиальное преобразование $\operatorname{sl}(2)$-системы Шлезингера фуксова типа. Строгие утверждение и доказательство будут приведены в следующем параграфе. 


\section{§3. $s l(2)$-связности с особенностями на $\mathbb{P}^{1}$}

Сначала опишем начальные данные нашей изомонодромной деформации, следуя [1]. Зафиксируем набор чисел $\left\{\lambda_{i}\right\}_{i=1}^{n}$ и модуль $\mathfrak{M}$ с носителем $S$, состоящим из различных точек $\left\{x_{i}\right\}_{i=1}^{n}$ на $\mathbb{P}^{1}$. Поскольку на $\mathbb{P}^{1}$ есть действие трехмерной группы автоморфизмов дробно-линейными преобразованиями, то разумно предполагать, что число точек $n$ больше или равно 3 . Рассмотрим векторное расслоение $\mathscr{L}$ ранга 2 на $\mathbb{P}^{1}$ с фиксированным детерминантным изоморфизмом $\phi: \bigwedge^{2} \mathscr{L} \simeq \mathscr{O}$ и логарифмическую связность с особенностями в $\mathfrak{M}$ и с собственными значениями вычета $\operatorname{Res}_{x_{i}} \nabla$, равными $\left(\lambda_{i},-\lambda_{i}\right)$. Кроме того, наложим на собственные значения условие

$$
\sum \epsilon_{i} \lambda_{i} \notin \mathbb{Z}, \quad\left(\epsilon_{1}, \ldots, \epsilon_{n}\right) \in(\mathbb{Z} / 2 \mathbb{Z})^{n},
$$

гарантирующее неразложимость нашей пары «расслоение со связностью» и, значит, ее стабильность. Таким образом, мы зафиксировали $s l(2)$-орбиты вычетов связности. Далее, обозначим собственные подпространства оператора $\operatorname{Res}_{x_{i}} \nabla$ через

$$
\ell_{i}^{ \pm}:=\operatorname{ker}\left(\operatorname{Res}_{x_{i}} \nabla \mp \lambda_{i}\right) .
$$

Для двух различных точек $x_{i}, x_{j} \in S$ рассмотрим модифицированное $S L(2)$ расслоение

$$
\mathscr{L}^{\prime \prime}=\left(x_{j}, \ell_{j}^{+}\right)^{\text {up }} \circ\left(x_{i}, \ell_{i}^{-}\right)^{\text {low }} \mathscr{L}
$$

с модифицированной $s l(2)$-связностью $\widetilde{\nabla}^{\prime \prime}$, определенной выше. Теперь определим операцию $(\downarrow \uparrow)_{i j}$ на $S L(2)$-расслоениях со связностями:

$$
(\downarrow \uparrow)_{i j}:(\mathscr{L}, \nabla) \mapsto\left(\mathscr{L}^{\prime \prime}, \nabla^{\prime \prime}+\omega_{i j}\right), \quad \omega_{i j}=\frac{1}{2}\left(\frac{d z}{z-x_{j}}-\frac{d z}{z-x_{i}}\right) .
$$

Другими словами, мы построили нетривиальные преобразования грубого пространства модулей $\mathscr{M}_{n}$. Такие преобразования являются изоморфизмами между пространствами $\mathscr{M}_{n}$ с различными наборами собственных значений вычетов связности. Вычислим, какие наборы собственных значений связности переходят друг в друга при изоморфизмах соответствующих пространств модулей.

ПРЕДЛОЖЕНИЕ 1. Модифицированная пара $\left(\mathscr{L}^{\prime \prime}, \widetilde{\nabla}^{\prime \prime}\right)$ является элементом грубого пространства модулей $\mathscr{M}_{n} ;$ собственные значения вычетов модифииированной связности $\operatorname{Res}_{x_{\alpha}} \widetilde{\nabla}^{\prime \prime}$ равны

$$
\left\{\lambda_{1}, \ldots, \lambda_{i}+\frac{1}{2}, \ldots, \lambda_{j}-\frac{1}{2}, \ldots, \lambda_{n}\right\}
$$

в случае пары модификащий в различных точках $x_{i}, x_{j} \in S u$

$$
\left\{\lambda_{1}, \ldots, \lambda_{k}+1, \ldots, \lambda_{n}\right\}
$$

в случае парь модификаций в одной точке $x_{k} \in S$.

ДокАЗАтЕльство. Первая часть предложения следует непосредственно из конструкции преобразования $(\uparrow \downarrow)_{i j}$. Сравним собственные значения вычетов связности $\nabla$ и модифицированной связности $\nabla^{\prime \prime}$ :

$$
\left\{\begin{array}{cc}
\lambda_{i} & \lambda_{j} \\
-\lambda_{i} & -\lambda_{j}
\end{array}\right\} \stackrel{\left(x_{i}, \ell_{i}^{-}\right)^{\mathrm{low}} \circ\left(x_{j}, \ell_{j}^{+}\right)^{\mathrm{up}}}{\longrightarrow}\left\{\begin{array}{cc}
\lambda_{i}+1 & \lambda_{j}-1 \\
-\lambda_{i} & -\lambda_{j}
\end{array}\right\} \stackrel{+\omega_{i j}}{\longrightarrow}\left\{\begin{array}{cc}
\lambda_{i}+1-\frac{1}{2} & \lambda_{j}-1+\frac{1}{2} \\
-\lambda_{i}-\frac{1}{2} & -\lambda_{j}+\frac{1}{2}
\end{array}\right\},
$$


и, таким образом, мы получаем сдвиги собственных значений

$$
\lambda_{i} \rightarrow \lambda_{i}+\frac{1}{2}, \quad \lambda_{j} \rightarrow \lambda_{j}-\frac{1}{2} .
$$

В случае пары модификаций в одной точке $x_{k}$

$$
\left(x_{k}, \ell_{k}^{-}\right)^{\text {low }} \circ\left(x_{k}, \ell_{k}^{-}\right)^{\text {up }}:\left\{\begin{array}{c}
\lambda_{k} \\
-\lambda_{k}
\end{array}\right\} \longrightarrow\left\{\begin{array}{c}
\lambda_{k}+1 \\
-\lambda_{k}-1
\end{array}\right\}
$$

1-форма $\omega_{k k}$ равна нулю, и поэтому мы получаем длинный сдвиг

$$
\lambda_{k} \rightarrow \lambda_{k}+1
$$

Такие модификации пар $(\mathscr{L}, \nabla)$ являются элементами бесконечного порядка дискретной аффинной группы преобразований соответствующего пространства модулей $\mathscr{M}_{n}$; при доказательстве предложения было, в частности, показано, что аффинная часть этой группы содержит короткие и длинные сдвиги. Конечная часть порождается перестановками точек в носителе модуля $\mathfrak{M}$ и сменами знаков собственных значений вычетов связности (локальными транспозициями группы Вейля).

Для описания группы преобразований ключевым является тот факт, что наша группа порождается отражениями, поскольку существует полная классификация таких групп, данная Г. Кокстером (см. [5]). Выберем удобные обозначения. Обозначим через $(i j)$ перестановку точек $x_{i}$ и $x_{j}$ носителя $S$ модуля $\mathfrak{M}$, а локальные перестановки группы Вейля $\mathfrak{W}\left(S L_{2}\right)$ в каждой точке $x_{i}$ обозначим через

$$
\sigma^{i}:\left(\begin{array}{cc}
\lambda_{i} & 0 \\
0 & -\lambda_{i}
\end{array}\right) \longrightarrow\left(\begin{array}{cc}
-\lambda_{i} & 0 \\
0 & \lambda_{i}
\end{array}\right)
$$

Теперь мы сопоставим нашей группе преобразований граф Kокстера типа $\widehat{C}_{n}$ и, таким образом, опишем эту группу с помощью классификации Кокстера. Конечная часть группы порождается элементами порядка два - отражениями; образующие представляются вершинами графа Кокстера, а соотношения ребрами.

1. Образующие группы $\mathfrak{S}_{n}$ суть перестановки $(i j)$, причем произведение двух соседних перестановок $(i j)(j k)=(i j k)$ является элементом третьего порядка. Такое соотношение между данными образующими можно представить в виде графа Кокстера $(i j) \longleftrightarrow(j k)$. Если же произведение двух отражений имеет порядок два, то условимся не соединять соответствующие вершины.

2. Образующие группы $(\mathbb{Z} / 2 \mathbb{Z})^{n}$ - локальные транспозиции $\sigma^{i}$; при этом композиция $\sigma^{i} \circ(i j)$ имеет порядок четыре. В этом случае граф Кокстера имеет вид $\sigma^{i} \Longleftrightarrow(i j)$.

3. Образующими аффинной части $T$ являются пары модификаций $(\uparrow \downarrow)_{i j}$ в соответствии с предложением, доказанным выше; как уже говорилось, точки $i$ и $j$, в которых производятся модификации, могут совпадать или не совпадать, и, таким образом, аффинные сдвиги могут быть короткими или длинными.

ПРЕДЛОЖЕНИЕ 2. Дискретная аффинная группа преобразований грубого пространства модулей $\mathscr{M}_{n}$ представляется в виде графа Кокстера типа $\widehat{C}_{n}$ $(\uparrow \downarrow)_{1} \circ \sigma^{1} \Rightarrow(\uparrow \downarrow)_{12} \circ(12) \leftrightarrow(\uparrow \downarrow)_{23} \circ(23) \leftrightarrow \cdots \leftrightarrow(\uparrow \downarrow)_{n-1, n} \circ(n-1 n) \Leftarrow(\uparrow \downarrow)_{n} \circ \sigma^{n}$ 
В следующих параграфах полученный результат иллюстрируется на частных примерах $\mathfrak{M}=x_{1}+x_{2}+x_{3}$ и $\mathfrak{M}=x_{1}+x_{2}+x_{3}+x_{4}$, которые соответствуют случаям гипергеометрического уравнения и уравнения Гойна. В классических работах К.-Ф. Гаусса, Э. Куммера и К. Гойна эти уравнения рассматривались в случае $G L(2)$, т. е. изучались $g l(2)$-связности. Наши вычисления можно легко обобщить на $g l(2)$-случай; обозначим через $\widetilde{\mathscr{M}}_{n}$ пространство модулей пар $(\mathscr{L}, \nabla)$ с фиксированными собственными значениями $\left(\mu_{i}, \nu_{i}\right)$ вычетов $\operatorname{Res}_{x_{i}} \nabla$. Изоморфизмы между пространствами модулей представляются теми же преобразованиями за исключением прибавления 1-формы $\omega_{i j}$, поскольку нам в этом случае нет необходимости следить за следом связности:

$$
\begin{aligned}
& \widetilde{\mathscr{M}}_{n}\left(\mathscr{L}, \nabla ;\left(\mu_{1}, \nu_{1}\right), \ldots,\left(\mu_{n}, \nu_{n}\right)\right) \\
& \simeq \widetilde{\mathscr{M}}_{n}\left(\mathscr{L}, \nabla ;\left(\mu_{1}, \nu_{1}\right), \ldots,\left(\mu_{i} \pm 1, \nu_{i}\right), \ldots,\left(\mu_{n}, \nu_{n}\right)\right), \\
& \widetilde{\mathscr{M}}_{n}\left(\mathscr{L}, \nabla ;\left(\mu_{1}, \nu_{1}\right), \ldots,\left(\mu_{i}, \nu_{i}\right), \ldots,\left(\mu_{n}, \nu_{n}\right)\right) \\
& \simeq \widetilde{\mathscr{M}}_{n}\left(\mathscr{L}, \nabla ;\left(\mu_{1}, \nu_{1}\right), \ldots,\left(\nu_{i}, \mu_{i}\right), \ldots,\left(\mu_{n}, \nu_{n}\right)\right), \\
& \widetilde{\mathscr{M}}_{n}\left(\mathscr{L}, \nabla ;\left(\mu_{1}, \nu_{1}\right), \ldots,\left(\mu_{i}, \nu_{i}\right), \ldots,\left(\mu_{j}, \nu_{j}\right), \ldots,\left(\mu_{n}, \nu_{n}\right)\right) \\
& \quad \simeq \widetilde{\mathscr{M}}_{n}\left(\mathscr{L}, \nabla ;\left(\mu_{1}, \nu_{1}\right), \ldots,\left(\mu_{j}, \nu_{j}\right), \ldots,\left(\mu_{i}, \nu_{i}\right), \ldots,\left(\mu_{n}, \nu_{n}\right)\right) .
\end{aligned}
$$

Также отметим, что последний изоморфизм, переставляющий вычеты связности, можно представлять на сфере Римана дробно-линейными преобразованиями. Поскольку группа дробно-линейных преобразований трехмерна, то в частных случаях трех и четырех точек такое представление особенно удобно для вычислений и мы им пользуемся.

\section{§4. Классический пример: $\mathfrak{W}\left(\widehat{C}_{3}\right)$-симметрии гипергеометрического уравнения}

Сначала напомним, каким образом мероморфные связности на $\mathbb{P}^{1}$ определяют системы дифференциальных уравнений с особенностями, и опишем нашу геометрическую конструкцию в терминах этих систем и их фундаментальных решений, следуя работе Болибруха [4]. Рассмотрим векторное расслоение $\mathscr{E}$ и ковариантную производную $\nabla_{v}$ для произвольного векторного поля $v$ с нулями в $\mathfrak{M}$. Тривиализуем на открытом множестве $U=\mathbb{P}^{1} \backslash S$ расслоение $\mathscr{E}$; в тривиальном расслоении имеется каноническая связность

$$
\left.\nabla_{v}\right|_{U} \simeq \partial_{v}
$$

являющаяся обыкновенной производной вдоль $v$. Для каждой особой точки $x_{i} \in S$ выберем ее окрестность $V_{i}$ и также тривиализуем расслоение $\mathscr{E}$, применив двойственность между векторными полями и 1-формами. Тогда на всей сфере Римана $\mathbb{P}^{1}$ получим $\nabla_{v}=\partial_{v}-\omega$, где 1-форма $\omega$ определяется векторным полем $v$. Нас интересует случай, когда особенности формы $\omega$ суть простые полюсы, и в этом случае тривиализующими отображениями в окрестностях $V_{i}$ будут отображения вычетов, инвариантные относительно выбора локальных параметров $z$; явное выражение для связности имеет вид

$$
\nabla=\frac{d}{d z}-\sum_{i} \frac{\operatorname{res}_{x_{i}} \omega}{z-x_{i}}
$$


Сечение $h(z)$ расслоения $\mathscr{E}$ называется горизонтальным относительно связности $\nabla$, если $\nabla_{v}(h) \equiv 0$, или, чуть подробнее, $\partial_{v} h=\omega h$. Таким образом, горизонтальные сечения относительно ковариантной производной вдоль векторного поля $\partial / \partial z$ задают систему дифференциальных уравнений

$$
d Y=\omega Y, \quad \text { где } \omega=B(z) d z .
$$

Производя замену базиса сечений

$$
Y^{\prime}=g Y, \quad \text { где } g \in G L(2) \otimes \mathbb{C}((z)),
$$

мы меняем матричную 1-форму $\omega$ :

$$
\omega^{\prime}=d g \cdot g^{-1}+g \cdot \omega \cdot g^{-1}
$$

такие преобразования системы называются калибровочными. Далее, рассмотрим действие монодромии на решения нашей системы: если $\gamma$ - контур, обходящий особую точку матрицы $B(z)$, то аналитическое продолжение вдоль $\gamma$ дает $Y \rightarrow Y \cdot g_{\gamma}$, где $g_{\gamma}-$ матрица монодромии. Предположим, что $z=0$ является особой точкой матрицы $B(z)$, а $\gamma$ - соответствующий элемент фундаментальной группы. Тогда поведение решения $Y(z)=\left(y_{1}(z), y_{2}(z)\right)$ нашей системы в окрестности особой точки $z=0$ описывается следующим образом.

$B$ окрестности особой точки $z=0$ фундаментальное решение $Y(z)$ имеет представление

$$
Y(z)=U(z) \cdot z^{A} \cdot z^{E},
$$

где $E:=(1 / 2 \pi \sqrt{-1}) \ln g_{\gamma}$, матрица $U(z)$ голоморфна и обратима в окрестности точки $z=0, A:=\operatorname{diag}\left(\sigma_{0}, \tau_{0}\right)$, а $\sigma_{0} u \tau_{0}-$ нормирования (показатели) компонент $y_{1}(z)$ и $y_{2}(z)$ в окрестности точки $z=0$ (нормированием решения $y(z)$ называется $\sup \left\{\left.k \in \mathbb{Z}|y(z) /| z\right|^{s} \rightarrow 0\right.$ для любого $s<k$ при $\left.\left.z \rightarrow 0\right\}\right)$.

Рассмотрим калибровочное преобразование специального вида

$$
g_{0}:=U(z)\left(\begin{array}{cc}
z & 0 \\
0 & 1
\end{array}\right) U(z)^{-1}
$$

его действие сдвигает нормирования решений:

$$
g_{0} \cdot Y(z)=U(z) z^{A^{\prime}} z^{E}, \quad \text { где } A^{\prime}=\left(\begin{array}{cc}
\sigma_{0} & 0 \\
0 & \tau_{0}
\end{array}\right)+\left(\begin{array}{ll}
1 & 0 \\
0 & 0
\end{array}\right) .
$$

Для произвольного простого полюса $x_{i}$ матрицы $B(z)$

$$
g_{i}=U\left(z-x_{i}\right)\left(\begin{array}{cc}
z-x_{i} & 0 \\
0 & 1
\end{array}\right) U\left(z-x_{i}\right)^{-1}
$$

или

$$
g_{i}=U\left(z-x_{i}\right)\left(\begin{array}{cc}
\left(z-x_{i}\right)^{-1} & 0 \\
0 & 1
\end{array}\right) U\left(z-x_{i}\right)^{-1} .
$$

Таким образом, пары модификаций наборов $(\mathscr{L}, \nabla)$ являются сингулярными калибровочными преобразованиями, и именно в таком виде модификации впервые появились в работах Биркгофа [3]. Сейчас мы убедимся, что они не меняют систему, а лишь репараметризуют ее. 
Рассмотрим дифференциальное уравнение второго порядка на $\mathbb{P}^{1}$ с простыми полюсами в трех различных точках $x_{1}, x_{2}, x_{3} \in \mathbb{P}^{1}$. Набор его решений можно задать в терминах схемы Римана

$$
\left(\begin{array}{lll}
x_{1} & x_{2} & x_{3} \\
\sigma_{1} & \sigma_{2} & \sigma_{3} \\
\tau_{1} & \tau_{2} & \tau_{3}
\end{array}\right)
$$

этого уравнения. Числа $\sigma_{i}, \tau_{i}$ являются показателями компонент решения в окрестности особой точки $x_{i}$. То есть если $y_{1}, y_{2}$ - набор линейно независимых решений в окрестности точки $z=a$, то

$$
y_{1} \sim(x-a)^{\sigma_{a}}, \quad y_{2} \sim(x-a)^{\tau_{a}} .
$$

Показатели удовлетворяют условию Фукса

$$
\sum_{\mathrm{i}=1}^{3}\left(\sigma_{i}+\tau_{i}\right)=1
$$

которое по сути является детерминантным изоморфизмом $\phi$ из $\S 3$ или, другими словами, формулой суммы вычетов для ковариантной производной, рассмотренной в начале этого параграфа. Известно, что в случае уравнений второго порядка схема Римана однозначно определяет дифференциальное уравнение (проблема Римана-Гильберта имеет положительное решение); для нашей схемы уравнение принимает вид

$$
\begin{aligned}
\frac{d^{2} y}{d z^{2}}+\left(\sum_{i=1}^{3} \frac{1-\sigma_{i}-\tau_{i}}{z-x_{i}}\right) \frac{d y}{d z} & \\
& +\left(\sum_{i=1}^{3} \frac{\sigma_{i} \tau_{i}\left(x_{i}-x_{i+1}\right)\left(x_{i}-x_{i+2}\right)}{z-x_{i}}\right) \frac{y}{\left(z-x_{1}\right)\left(z-x_{2}\right)\left(z-x_{3}\right)}=0
\end{aligned}
$$

(подразумевается, что $x_{4}=x_{1}$ и $x_{5}=x_{2}$ ).

Можно считать, что особые точки $x_{1}, x_{2}, x_{3}$ - это $0,1, \infty$, и соответствующими дробно-линейными заменами свести такое уравнение к гипергеометрическому уравнению

$$
z(1-z) \frac{d^{2} y}{d z^{2}}+[\gamma-(\alpha+\beta+1) z] \frac{d y}{d z}-\alpha \beta \cdot y=0
$$

параметры уравнения $\alpha, \beta, \gamma \in \mathbb{C} \backslash\{-1,-2, \ldots\}$ связаны с показателями схемы Римана и удовлетворяют соотношению Фукса. На языке схем Римана это преобразование означает приведение схемы к наиболее простому виду:

$$
\left(\begin{array}{ccc}
0 & 1 & \infty \\
\sigma_{0} & \sigma_{1} & \sigma_{\infty} \\
\tau_{0} & \tau_{1} & \tau_{\infty}
\end{array}\right)=z^{\sigma_{0}}(z-1)^{\sigma_{1}}\left(\begin{array}{ccc}
0 & 1 & \infty \\
0 & 0 & \sigma_{\infty}+\sigma_{0}+\sigma_{1} \\
\tau_{0}-\sigma_{0} & \tau_{1}-\sigma_{1} & \tau_{\infty}+\sigma_{0}+\sigma_{1}
\end{array}\right) .
$$

Решение гипергеометрического уравнения с показателем 0 в точке $z=0$ представляется всюду сходящимся рядом

$$
F(\alpha, \beta, \gamma \mid z)=\sum_{n} \frac{(\alpha)_{n}(\beta)_{n}}{(\gamma)_{n}} \cdot \frac{z^{n}}{n !}
$$


где $(a)_{n}:=\Gamma(a+n) / \Gamma(a)$, а $\Gamma(x)$ - гамма-функция Эйлера, и называется гипергеометрической функцией. Далее мы исследуем симметрии уравнения и представляем их в виде соотношений на гипергеометрические функции; затем сопоставляем эти вычисления с результатом, полученным в предыдущем параграфе.

По виду гипергеометрической функции и уравнения сразу же можно заметить симметрию $\beta \rightleftarrows \alpha$. Далее, поскольку схема Римана гипергеометрического уравнения имеет вид

$$
\left(\begin{array}{ccc}
0 & 1 & \infty \\
0 & 0 & \alpha \\
1-\gamma & \gamma-\alpha-\beta & \beta
\end{array}\right)
$$

то в нуле имеется два независимых решения с показателями 0 и $1-\gamma$,

$$
y_{0}^{\text {hol }}=F(\alpha, \beta, \gamma \mid z) \quad \text { и } \quad y_{0}^{1-\gamma}=z^{1-\gamma} F(\alpha+1-\gamma, \beta+1-\gamma, 2-\gamma \mid z) \text {; }
$$

первое из них голоморфное, а второе имеет монодромию $e^{2 \pi \sqrt{-1}} e^{-2 \pi \sqrt{-1} \gamma}$ при обходе вокруг нуля. Точки 0,1 и $\infty$ можно переставлять преобразованиями Мёбиуса

$$
(01): z \rightarrow 1-z, \quad(0 \infty): z \rightarrow \frac{1}{z} \quad \text { и } \quad(1 \infty): z \rightarrow \frac{z}{z-1}
$$

и из решений в нуле получить решения в остальных точках:

$$
\begin{aligned}
y_{1}^{\text {hol }} & =F(\alpha, \beta, \alpha+\beta+1-\gamma \mid 1-z), \\
y_{1}^{\gamma-\alpha-\beta} & =(1-z)^{\gamma-\alpha-\beta} F(\gamma-\alpha, \gamma-\beta, \gamma-\alpha-\beta+1 \mid 1-z), \\
y_{\infty}^{\alpha} & =z^{-\alpha} F(\alpha, \alpha+1-\gamma, \alpha+1-\beta \mid 1 / z), \\
y_{\infty}^{-\beta} & =z^{-\beta} F(\beta, \beta+1-\gamma, \beta+1-\alpha \mid 1 / z) .
\end{aligned}
$$

Для каждого решения существует четыре представления; различные выражения этих решений называются двадцатью четырьмя соотношениями Куммера (см. [2]).

Теперь рассмотрим действие модификаций, причем не будем прибавлять 1 -форму $\omega_{i j}$. Однако мы сохраним прежние обозначения ввиду удобства. Например, пара модификаций $(\uparrow \downarrow)_{1 \infty}$ имеет вид

$$
\left(\begin{array}{ccc}
0 & 1 & \infty \\
0 & 0 & \alpha \\
1-\gamma & \gamma-\alpha-\beta & \beta
\end{array}\right) \longrightarrow\left(\begin{array}{ccc}
0 & 1 & \infty \\
0 & 0 & \alpha+1 \\
1-\gamma & \gamma-\alpha-\beta-1 & \beta
\end{array}\right)
$$

что соответствует сдвигу $\alpha \rightarrow \alpha+1$. Сдвиг параметра $\alpha$ не меняет гипергеометрическое уравнение, т. е. соответствующие схемы Римана эквивалентны:

$$
\left(\begin{array}{ccc}
0 & 1 & \infty \\
0 & 0 & \alpha \\
1-\gamma & \gamma-\alpha-\beta & \beta
\end{array}\right) \approx\left(\begin{array}{ccc}
0 & 1 & \infty \\
0 & 0 & \alpha+1 \\
1-\gamma & \gamma-\alpha-\beta-1 & \beta
\end{array}\right)
$$

Действительно,

а значит

$$
\alpha z^{\alpha-1} F(\alpha+1, \beta, \gamma)=\frac{\partial}{\partial z} z^{\alpha} F(\alpha, \beta, \gamma)
$$

$$
F(\alpha+1)=F(\alpha)+\frac{z}{\alpha} \frac{\partial}{\partial z} F(\alpha)
$$


для $\alpha^{\prime}:=\alpha+1-\gamma$ аналогичным образом получаем

$$
\alpha^{\prime} z^{\alpha^{\prime}-1} F\left(\alpha^{\prime}+1\right)=\alpha^{\prime} z^{\alpha-\gamma} F\left(\alpha^{\prime}\right)+z^{\alpha^{\prime}} F^{\prime}\left(\alpha^{\prime}\right) .
$$

Таким образом, для второго независимого решения $F_{2}\left(\alpha^{\prime}\right):=z^{1-\gamma} F(\alpha+1-\gamma)$

$$
F_{2}\left(\alpha^{\prime}+1\right)=\frac{\alpha}{\alpha^{\prime}} F_{2}\left(\alpha^{\prime}\right)+\frac{z}{\alpha^{\prime}} F_{2}^{\prime}\left(\alpha^{\prime}\right) .
$$

Окончательно,
$\left\{\begin{array}{c}F(\alpha+1, \beta, \gamma \mid z) \\ z^{1-\gamma} F(\alpha+1-\gamma+1, \beta+1-\gamma, 2-\gamma \mid z)\end{array}\right\}=\left\{\begin{array}{c}F(\alpha, \beta, \gamma \mid z)+\frac{z}{\alpha} \frac{\partial}{\partial z} F(\alpha, \beta, \gamma \mid z) \\ \frac{\alpha}{\alpha+1-\gamma} F_{2}+\frac{z}{\alpha+1-\gamma} \frac{\partial}{\partial z} F_{2}\end{array}\right\} ;$

это и является соотношением Гаусса. Таким образом «модифицированные» решения $\dot{y}_{1, \infty}(\alpha-1)$ выражаются через «немодифицированные» $\dot{y}_{1, \infty}(\alpha)$ и их производные $(\partial / \partial z) y_{1, \infty}(\alpha)$; поэтому после подходящей замены гипергеометрическое уравнение перейдет в себя. Как и раньше, модификации порождают аффинные симметрии уравнения. В результате 24 соотношения Куммера вместе с соотношением Гаусса и с очевидной симметрией $\sigma^{\infty}: \alpha \rightleftarrows \beta$ порождают группу дискретных симметрий дифференциального уравнения второго порядка с тремя простыми полюсами в точках $x_{1}, x_{2}, x_{3}$ на $\mathbb{P}^{1}$. Структура группы описывается следующим $\widehat{C}_{3}$-графом Кокстера:

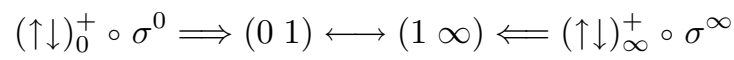

Поясним групповые соотношения в терминах схемы Римана:

$$
\begin{gathered}
(\uparrow \downarrow)_{0}^{+}:\left(\begin{array}{ccc}
0 & 1 & \infty \\
0 & 0 & \alpha \\
1-\gamma & \gamma-\alpha-\beta & \beta
\end{array}\right) \longrightarrow\left(\begin{array}{ccc}
0 & 1 & \infty \\
0+1 & 0 & \alpha \\
1-\gamma-1 & \gamma-\alpha-\beta & \beta
\end{array}\right) \\
=z\left(\begin{array}{ccc}
0 & 1 & \infty \\
0 & 0 & \alpha+1 \\
1-\gamma-2 & \gamma-\alpha-\beta & \beta+1
\end{array}\right), \quad \text { т. e. }\left\{\begin{array}{l}
\alpha \rightarrow \alpha+1 \\
\beta \rightarrow \beta+1 \\
\gamma \rightarrow \gamma+2
\end{array}\right\}, \\
(\uparrow \downarrow)_{\infty}^{-}:\left(\begin{array}{ccc}
0 & 1 & \infty \\
0 & 0 & \alpha \\
1-\gamma & \gamma-\alpha-\beta & \beta
\end{array}\right) \rightarrow\left(\begin{array}{ccc}
0 & 1 & \infty \\
0 & 0 & \alpha+1 \\
1-\gamma & \gamma-\alpha-\beta & \beta-1
\end{array}\right), \quad\left\{\begin{array}{c}
\alpha \rightarrow \alpha+1 \\
\beta \rightarrow \beta-1 \\
\gamma \rightarrow \gamma
\end{array}\right\} .
\end{gathered}
$$

При помощи соотношений Куммера и Гаусса аналогично проверяются остальные соотношения в группе.

\section{§5. Другой классический пример: $\mathfrak{W}\left(\widehat{C}_{4}\right)$-симметрии уравнения Гойна}

Следующим шагом нашего рассмотрения будет уравнение Гойна. Всякое фуксово уравнение второго порядка с четырьмя особыми точками эквивалентно уравнению Гойна

$$
\frac{d^{2} y}{d z^{2}}+\left[\frac{\gamma}{z}+\frac{\delta}{z-1}+\frac{\epsilon}{z-a}\right] \frac{d y}{d z}+\frac{\alpha \beta z-q}{z(z-1)(z-a)} y=0
$$

соотношение Фукса на параметры - это

$$
\alpha+\beta-\gamma-\delta+1-\epsilon=0 .
$$


Предполагается, что особыми точками являются $0,1, a, \infty$ и схема Римана уравнения имеет вид

$$
\left(\begin{array}{cccc|c}
0 & 1 & a & \infty & \\
0 & 0 & 0 & \alpha & q \\
1-\gamma & 1-\delta & 1-\epsilon & \beta &
\end{array}\right)
$$

где $q$ - вспомогательный параметр. Как и в случае гипергеометрического уравнения, схему Римана можно упростить при помощи дробно-линейных преобразований:

$$
\begin{aligned}
& \left(\begin{array}{cccc}
0 & 1 & a & \infty \\
\sigma_{0} & \sigma_{1} & \sigma_{a} & \sigma_{\infty} \\
\tau_{0} & \tau_{1} & \tau_{a} & \tau_{\infty}
\end{array} \mid q\right) \\
& =z^{\sigma_{0}}(z-1)^{\sigma_{1}}(z-a)^{\sigma_{a}}\left(\begin{array}{cccc|c}
0 & 1 & a & \infty \\
0 & 0 & 0 & \sigma_{\infty}+\sigma_{0}+\sigma_{1}+\sigma_{a} & q^{\prime} \\
\tau_{0}-\sigma_{0} & \tau_{1}-\sigma_{1} & \tau_{a}-\sigma_{a} & \tau_{\infty}+\sigma_{0}+\sigma_{1}+\sigma_{a} &
\end{array}\right) .
\end{aligned}
$$

Следует отметить, что в случае четырех особых точек уже нет взаимно однозначного соответствия между схемами Римана и уравнениями фуксова типа, и именно для этого вводится вспомогательный параметр q. При преобразованиях уравнения необходимо следить за его изменениями наряду с изменениями схемы Римана. Перестановки особых точек порождаются следующими транспозициями:

$$
z \rightarrow 1-z, \quad z \rightarrow \frac{1}{z}, \quad z \rightarrow \frac{a}{z}, \quad z \rightarrow z-a, \quad z \rightarrow \frac{1-a}{z-a}
$$

всего транспозиций такого вида двадцать четыре - по шесть возможных значений для каждой точки. Так, возможные значения $a-$ это $a, 1-a, a^{-1}$, $(1-a)^{-1}, a(a-1)^{-1},(a-1) a^{-1}$. Следуя К. Гойну, обозначим голоморфное решение в нуле через $y_{0}^{\text {hol }}=F(a|\alpha, \beta, \gamma, \delta| z)$ по аналогии с гипергеометрической функцией. Оно также допускает симметрию $\sigma^{\infty}: \alpha \rightleftarrows \beta$. Второе независимое неголоморфное в нуле решение - это

$$
y_{0}^{1-\gamma}=z^{1-\gamma} F(a|\alpha+1-\gamma, \beta+1-\gamma, \gamma, \delta| z)
$$

Действуя транспозициями, переставляющими особенности уравнения, на эту пару решений в нуле (и на схему Римана), получаем локальные решения в остальных особых точках:

$$
\begin{aligned}
y_{1}^{\text {hol }} & =F(1-a|\alpha, \beta, \gamma, \delta| 1-z), \\
y_{1}^{1-\delta} & =(z-1)^{1-\delta}(z-a)^{\alpha} F\left(a|\alpha+1-\delta, \gamma+1-\beta, \delta, 2-\gamma| \frac{a(z-1)}{z-a}\right), \\
y_{a}^{\text {hol }} & =F\left(\frac{1-a}{a}|\alpha, \beta, \alpha+\beta-\gamma-\delta+1, \delta, 2-\gamma| \frac{a-x}{a}\right), \\
y_{a}^{1-\epsilon} & =(x-1)^{\gamma+\delta-\alpha-\beta} F\left(\frac{1}{1-a}|\gamma+\delta-\beta, \gamma+\delta-\alpha, \delta, \gamma+\delta-\alpha-\beta+1| \frac{x-1}{x-a}\right), \\
y_{\infty}^{\alpha} & =z^{\alpha} F\left(\frac{1}{a}|\alpha, \alpha+1-\gamma, \beta+1-\alpha, \delta| \frac{1}{z}\right), \\
y_{\infty}^{\beta} & =z^{\beta} F\left(\frac{1}{a}|\beta, \beta+1-\gamma, \beta+1-\alpha, \delta| \frac{1}{z}\right) .
\end{aligned}
$$


С учетом шести выражений для каждой точки получается всего $8 \times 24=192$ подобных соотношения, аналогичных двадцати четырем соотношениям Куммера в гипергеометрическом случае. Таким образом, мы вычислили конечную часть группы симметрий порядка

$$
\left|\mathfrak{W}\left(C_{4}\right)\right|=\left|(\mathbb{Z} / 2 \mathbb{Z})^{4} \rtimes \mathfrak{S}_{4}\right|=192 \times 2
$$

с учетом транспозиции $\sigma^{\infty}$. Чтобы вычислить аффинную часть этой группы, нам потребуются соотношения между «модифицированными» и «немодифицированными» решениями $F(a \mid z)$, аналогичные соотношениям Гаусса в гипергеометрическом случае; они имеют следующий вид:

$$
\begin{aligned}
{\left[(\epsilon-1)-\frac{\alpha \beta}{\gamma} q\right] } & F\left(a, q^{\prime}|\alpha, \beta, \gamma+1, \delta+1| z\right) \\
& =(\epsilon-1) F(a, q|\alpha, \beta, \gamma, \delta| z)+(z-a) \frac{\partial}{\partial z} F(a, q|\alpha, \beta, \gamma, \delta| z),
\end{aligned}
$$

где $q^{\prime}$ - модифицированный вспомогательный параметр,

$$
q^{\prime}=q+a \frac{\gamma+\delta}{\alpha \beta}-\frac{\gamma}{\alpha \beta}
$$

объяснение модификации вспомогательного параметра будет приведено в следующем параграфе с использованием более удобной геометрической интерпретации пространства начальных данных уравнения Гойна - вспомогательный параметр $q$ имеет смысл обратного импульса в гамильтоновом представлении изомонодромной деформации уравнения Гойна.

Таким образом, можно подходящими заменами с учетом изменения вспомогательного параметра привести уравнение к исходному виду. Производная второго локального решения в нуле имеет вид

$$
\begin{array}{r}
\frac{\partial}{\partial z} z^{1-\gamma} F(\alpha+1-\gamma, \beta+1-\gamma, \gamma, \delta)=(1-\gamma) z^{-\gamma} F(\alpha+1-\gamma, \beta+1-\gamma, \gamma, \delta) \\
+z^{1-\gamma} F^{\prime}(\alpha+1-\gamma, \beta+1-\gamma, \gamma, \delta) .
\end{array}
$$

В результате

$$
\begin{gathered}
\left\{\begin{array}{c}
F\left(q^{\prime}|\alpha, \beta, \gamma+1, \delta+1| z\right) \\
z^{1-\gamma} F\left(q^{\prime}|\alpha+1-\gamma-1, \beta+1-\gamma-1, \gamma+1, \delta+1| z\right)
\end{array}\right\} \\
=\left\{\begin{array}{c}
\frac{\epsilon-1}{\epsilon-1-\frac{\alpha \beta}{\gamma} q} F(q|\alpha, \beta, \gamma, \delta| z)+\frac{z-a}{\epsilon-1-\frac{\alpha \beta}{\gamma} q} \frac{\partial}{\partial z} F(q|\alpha, \beta, \gamma, \delta| z) \\
\frac{\epsilon-1-\left(1-\frac{a}{z}\right)(1-\gamma)}{\epsilon-1-\frac{\alpha \beta}{\gamma} q} F_{2}+\frac{z-a}{\epsilon-1-\frac{\alpha \beta}{\gamma} q} \frac{\partial}{\partial z} F_{2}
\end{array}\right\},
\end{gathered}
$$

где $F_{2}=z^{1-\gamma} F(q|\alpha+1-\gamma, \beta+1-\gamma, \gamma, \delta| z)$. Комбинируя эти соотношения с действием дробно-линейных преобразований, получаем эквивалентность соответствующих схем Римана. Как объяснялось в §3, комбинация перестановки точек с парой модификаций определяет структуру полупрямого произведения на группе $\mathfrak{W}\left(\widetilde{C}_{4}\right) \simeq T \rtimes\left((\mathbb{Z} / 2 \mathbb{Z})^{4} \rtimes \mathfrak{S}_{4}\right)$. Таким образом, мы описали и проверили образующие и соотношения группы дискретных симметрий уравнения Гойна. 


\section{§6. Изомонодромная деформация уравнения Гойна - шестое уравнение Пенлеве}

И наконец, завершая обзор классических результатов, рассмотрим изомонодромную деформацию фуксова дифференциального уравнения второго порядка с четырьмя особенностями. Существует также другой подход к этой же задаче. Будем говорить, что дифференциальное уравнение обладает свойством Пенлеве, если оно не имеет подвижных точек ветвления и существенных особенностей - только подвижные полюсы. Шестое уравнение Пенлеве $P_{\mathrm{VI}}$ является самым общим дифференциальным уравнением второго порядка с не более чем четырьмя особенностями на $\mathbb{P}^{1}$, обладающим свойством Пенлеве.

Как и в предыдущем параграфе предполагаем, что особыми являются точки $0,1, t, \infty$. Уравнение $P_{\mathrm{VI}}$ имеет следующий вид:

$$
\begin{aligned}
\frac{d^{2} x}{d t^{2}}=\frac{1}{2}\left(\frac{1}{x}+\right. & \left.\frac{1}{x-1}+\frac{1}{x-t}\right)\left(\frac{d x}{d t}\right)^{2}-\left(\frac{1}{t}+\frac{1}{t-1}+\frac{1}{x-t}\right) \frac{d x}{d t} \\
& +\frac{x(x-1)(x-t)}{t^{2}(t-1)^{2}}\left(\alpha-\beta \frac{t}{x^{2}}+\gamma \frac{t-1}{(x-1)^{2}}+\left(\frac{1}{2}-\delta\right) \frac{t(t-1)}{(x-t)^{2}}\right) .
\end{aligned}
$$

Временем (параметром деформации) является двойное отношение особых точек $[0,1, t, \infty]$. Параметры уравнения $\alpha, \beta, \gamma, \delta$ соответствуют собственным значениям вычетов логарифмической связности следующим образом:

$$
\alpha=\frac{1}{2} \lambda_{\infty}^{2}, \quad \beta=\frac{1}{2} \lambda_{0}^{2}, \quad \gamma=\frac{1}{2} \lambda_{1}^{2}, \quad \delta=\frac{1}{2} \lambda_{t}^{2} .
$$

Нам будет удобно пользоваться гамильтоновой формой

$$
\left\{\begin{array}{l}
\frac{d x}{d t}=\frac{\partial H}{\partial p} \\
\frac{d p}{d t}=-\frac{\partial H}{\partial x}
\end{array}\right.
$$

изомонодромной системы $P_{\mathrm{VI}}$ с гамильтонианом

$$
\begin{aligned}
H=\frac{1}{t(t-1)} & {\left[x(x-1)(x-t) p^{2}\right.} \\
& \left.-\left\{\lambda_{0}(x-1)(x-t)+\lambda_{1} x(x-t)+\left(\lambda_{t}-1\right) x(x-1)\right\} p+\lambda(x-t)\right],
\end{aligned}
$$

где $\lambda=\frac{1}{4}\left[\left(\lambda_{0}+\lambda_{1}+\lambda_{t}-1\right)^{2}-\lambda_{\infty}^{2}\right]$.

Группа симметрий пространства модулей $\mathscr{M}_{4}$ содержит подгруппу $\mathfrak{W}\left(\widehat{D}_{4}\right)$, порожденную перестановками особых точек (а значит, и параметров $\alpha, \beta, \gamma, \delta$ ) и парами модификаций в различных точках (только «короткими» сдвигами). Действие такой группы вполне естественно в описанной ниже геометрической интерпретации [1].

Пространство начальных данных для изомонодромной системы $P_{\mathrm{VI}}$ является некомпактной комплексной поверхностью

$$
\left\{(x, p) \mid(x, p)^{\text {up }}: \mathscr{O} \oplus \mathscr{T}(-\mathfrak{M}) \rightarrow \mathscr{O} \oplus \mathscr{O}(-t)\right\}
$$

локально изоморфной $\mathscr{T}^{*}\left(\mathbb{P}^{1} \backslash S\right)$. Структура этой поверхности была изучена в работах [7] (см. также [2]) и [1]. Переменная «импульс» $p$ имеет смысл направ- 
ления верхней модификации в расслоении $(\mathscr{O} \oplus \mathscr{T}(-\mathfrak{M}))$ в точке $x$. В матричном представлении

$$
(x, p)^{\text {up }}=\left(\begin{array}{cc}
\omega & \eta \\
1 & 0
\end{array}\right),
$$

где $\omega \in \operatorname{Hom}(\mathscr{T}(-\mathfrak{M}), \mathscr{O}) \simeq \Omega(\mathfrak{M})$, а $\eta \in \Omega(\mathfrak{M}-t)$. Детерминант $\operatorname{det}(x, p)^{\text {up }}$ имеет простой нуль в точке $x$; поэтому

$$
\eta(x)=0, \quad \omega(x)=p d x .
$$

Эта процедура разделения переменных для $s l(2)$-системы Шлезингера с $n$ особенностями впервые появилась в работе Склянина [21].

Заметим, что пара модификаций, например в точках 0 и 1, меняет переменную «импульс» следующим образом:

$$
p \rightarrow p+\Delta p, \quad \Delta p=\frac{1}{x-1}-\frac{1}{x}
$$

(при этом $x$ не меняется), и сохраняет гамильтониан. Действительно,

$$
\begin{aligned}
H^{\prime \prime}=\frac{1}{t(t-1)} & {\left[x(x-1)(x-t)(p+\Delta p)^{2}-\left\{\left(\lambda_{0}+\frac{1}{2}\right)(x-1)(x-t)\right.\right.} \\
& \left.\left.+\left(\lambda_{1}-\frac{1}{2}\right) x(x-t)+\left(\lambda_{t}-1\right) x(x-1)\right\}(p+\Delta p)+\lambda(x-t)\right] \\
=H+2 p & \frac{1}{2}(x-t)+\frac{1}{4} \frac{x-t}{x(x-1)} \\
& -\frac{1}{2}\left[\lambda_{0} \frac{x-t}{x}+\lambda_{1} \frac{x-t}{x-1}+\left(\lambda_{t}-1\right)\right]-\frac{1}{2}(x-t)-\frac{1}{2} \frac{1}{2} \frac{x-t}{x(x-1)}=H .
\end{aligned}
$$

Полученную группу $\widehat{D}_{4}$-симметрий можно расширить с помощью группы $\mathfrak{S}_{3}$ автоморфизмов графа $D_{4}$ и получить аффинную группу $\mathfrak{W}\left(\widehat{F}_{4}\right)$ симметрий уравнения $P_{\mathrm{VI}}$, которая содержит полученную нами в общем случае группу $\mathfrak{W}\left(\widehat{C}_{4}\right)$. Таким образом, случай четырех точек является исключительным по размеру группы симметрий из-за наличия дополнительных автоморфизмов графа Кокстера.

\section{ЛитерАТУРА}

1. Arinkin D., Lysenko $S$. Isomorphisms between moduli of $S L(2)$-bundles with connections on $\mathbb{P}^{1} \backslash\left\{x_{1}, \ldots, x_{4}\right\}$. Math. Res. Lett., 4, 181-190 (1997).

2. Бейтмен Г., Эрдейи А. Высшие трансцендентные функции. тт. 1, 3, М., Наука, 1966.

3. Birkhoff G. D. Collected mathematical papers. Vol. I, Amer. Math. Soc., 1950.

4. Болибрух A. A. 21-я проблема Гильберта для фуксовых линейных систем. Труды МИРАН, 206, 1994.

5. Coxeter H. S. M., Moser W. O. J. Generators and relations for discrete groups. Springer-Verlag, 1972.

6. Дринфельд В. Г. Доказательство глобальной гипотезы Ленглендса для $G L(2)$ над функциональным полем. Функц. анализ и его прил., 11, вып. 3, 74-75 (1977).

7. Erdélyi A. Certain expansions of solutions of the Heun equation. Quart. J. Math., Oxford Ser., 15, 62-69 (1944). 
8. Enriquez B., Rubtsov V. Hecke-Tyurin parametrization of the Hitchin and KZB systems. arXiv: AG/9911087.

9. Iwasaki K., Kimura H., Shimomura S., Yoshida M. From Gauss to Painlevé. A modern theory of special functions. Aspects of Mathematics, E16. Friedr. Vieweg \& Sohn, Braunschweig, 1991.

10. Hecke E. Mathematische Werke. Göttingen, 1953.

11. Heun K. Zur Theorie Riemann'schen Functionen zweiter Ordnung mit vier Verzweigungspunkten. Math. Ann., XXXIII (1889).

12. Hitchin N. Twistor spaces, Einstein metrics and isomonodromic deformations. J. Diff. Geom., 3, 52-134, 1995.

13. Ince E. L. Ordinary differential equations. Oxford Univ. Press, 1953.

14. Кричевер И. М. Интегрирование нелинейных уравнений методами алгебраической геометрии. Функц. анализ и его прил., 11, вып. 1, 15-31 (1977).

15. Jimbo M., Miwa T. Monodromy preserving deformation of linear ordinary differential equations with rational coefficients. II. Physica 2D, 8, 407-448 (1981).

16. Korotkin D., Manojlović N., Samtleben H. Schlesinger transformations for elliptic isomonodromic deformations. J. Math. Phys., 41, 3125-3141 (2000).

17. Levin A. M., Olshanetsky M. A., Zotov A. Hitchin systems - symplectic Hecke correspondence and two-dimensional version. Comm. Math. Phys., 236, 93-133 (2003).

18. Okamoto $K$. Studies in the Painlevé equations I. Sixth Painlevé equation PVI. Ann. Mat. Pura Appl., 146, 337-381 (1987).

19. Schlesinger L. Über eine Klasse von Differentialsystemen beliebiger Ordnung mit festen kritischen Punkten. J. Reine Angew. Math., 141, 96-145 (1912).

20. Серр Ж.-П. Алгебраические группы и поля классов. Мир, М., 1968.

21. Склянин E. K. Разделение переменных в системе Годена. Зап. научн. сем. ЛОМИ, 164, 151-169 (1987).

22. Vakulenko $V$. Note on the Ruijsenaars-Schneider model. arXiv: QA/9909079.

23. Weil A. Généralisation des fonctions abéliennes. J. Math. Pures Appl., 17, 47-87 (1938).

Независимый московский университет, Московский физико-технический институт Поступило в редакцию e-mail: oblezin@mccme.ru 28 ноября 2002 г. 